\title{
STRONG-PRINCIPAL BIMODULES OF NEST ALGEBRAS
}

\author{
WEIBANG GONG AND JUN ZHU
}

(Communicated by Palle E. T. Jorgensen)

\begin{abstract}
We shall show that $B(H)$ can be represented by the strong closure of the linear span of the compounds of a fixed operator in $B(H)$ and the rank one operators, composed only by the vectors of a certain orthonormal basis of $H$, in a nest algebra, even, under some assumption, in the radical of a nest algebra.
\end{abstract}

\section{NOTATIONS, BASIC RELATIONSHIPS, AND INTRODUCTION}

Throughout, the nonzero Hilbert space $H$ under consideration is complex and separable. Subspace means "closed subspace of $H$ " and operator means "bounded linear operator from $H$ into itself." $\subseteq$ is used for "is contained in," while $\subset$ is reserved for "is properly contained in." We write $B(H)$ for the set of all operators and $K(H)$ for the set of all compact operators. The symbol $x \otimes y$ denotes the rank-1 operator $(\cdot, x) y$ for $x, y \in H$. If $S \subseteq H$ and $\mathscr{S} \subseteq \mathbf{B}(H)$, we write $R^{1}(S)$ for the set of all rank-1 operators composed by vectors in $S$ and $R_{S}^{1} \mathscr{S}$ and $R^{1} \mathscr{S}$ for $R^{1}(S) \cap \mathscr{S}$ and $R^{1}(H) \cap \mathscr{S}$ respectively. If $M$ is a subspace, $P_{M}$ stands for the projection from $H$ onto $M$. If $\mathscr{S} \subseteq$ $B(H),[\mathscr{S}]^{(s)}\left([\mathscr{S}]^{(n)}\right)$ denotes the strong closure (norm closure) of the linear span of $\mathscr{S}$. If $\mathscr{S}, \mathscr{A} \subseteq B(H)$, and if there exists $G$ in $B(H)$ such that $\mathscr{S}=[\mathscr{A} G \mathscr{A}]^{(s)}\left(\mathscr{S}=[\mathscr{A} G \mathscr{A}]^{(n)}\right)$, then we call $\mathscr{S}$ a strong-principal (normprincipal) bimodule of $\mathscr{A}$ with respect to $G$.

A nest $\mathscr{N}$ is a family of subspaces totally ordered by inclusion. $\mathscr{N}$ is said to be complete if (i) it contains $\{0\}$ and $H$ and (ii) given any subfamily $\mathscr{N}_{0}$ of $\mathcal{N}$, the subspaces $\Lambda\left\{L: L \in \mathscr{N}_{0}\right\}$ and $\bigvee\left\{L: L \in \mathscr{N}_{0}\right\}$ are both members of $\mathscr{N}$. If $N \in \mathscr{N}$, we define $N_{-}=\bigvee\{L: L \subset N, L \in \mathscr{N}\}$ and $N_{+}=$ $\bigwedge\{L: N \subset L, L \in \mathscr{N}\}$. Obviously, if $\mathscr{N}$ is complete, then $N_{-}, N_{+} \in \mathscr{N}$ for all $N \in \mathscr{N}$. Throughout, a nest is complete. The set $\{T: T \in B(H), T N \subseteq N$, $N \in \mathscr{N}\}$ is called the nest algebra (associated with $\mathscr{N}$ ) and is denoted in this paper by $A \mathcal{N}$. The space $M \ominus N$, with $M, N \in \mathscr{N}$ and $M \supseteq N$, is called an $\mathscr{N}$-interval. A finite $\mathscr{N}$-partition is a finite set $\left\{E_{1}, \ldots, E_{n}\right\}$ of mutually orthogonal $\mathscr{N}$-intervals with $E_{1} \oplus \cdots \oplus E_{n}=H$. The Jacobson radical [5, p. 69] of $A \mathscr{N}$ is denoted by $R A \mathscr{N}$. We can regard the following theorem as another

Received by the editors December 5, 1990.

1980 Mathematics Subject Classification (1985 Revision). Primary 47A65, 47C65, 47D15.

Key words and phrases. Bimodule, rank-1 operator, nest algebra. 
definition of the radical, which is actually used in this paper.

Theorem. (Ringrose Criterion, [5, p. 73]). Let $\mathcal{N}$ be a complete nest. If $T \in A \mathscr{N}$, then $T \in R A \mathcal{N}$ if and only if for each $\varepsilon>0$ there exists a finite $\mathscr{N}$-partition $\left\{E_{n}\right\}$ such that $\left\|P_{E_{n}} T P_{E_{n}}\right\|<\varepsilon$ for each $n$.

Corollary. [4, p. 21]. Let $\mathscr{N}$ be a complete nest. Then

$$
R A \mathscr{N}=\left[\left\{P_{N} T P_{N^{\perp}}: N \in \mathscr{N}, T \in B(H)\right\}\right]^{(n)} .
$$

Definition. (Larson, [3, p.418]). Let $\mathscr{N}$ be a complete nest. The symbol $R^{\infty} A \mathscr{N}$ is defined to be the class of all operators $T$ in $A \mathscr{N}$ with the property that given $\varepsilon>0$ there exists a (perhaps infinite) $\mathscr{N}$-partition $\left\{E_{n}\right\}$ with $\left\|P_{E_{n}} T P_{E_{n}}\right\|<\varepsilon$ for each $n$.

Obviously, $R A \mathscr{N} \subseteq R^{\infty} A \mathcal{N}$. Finally, the following operator $G$ will play an important role in this paper. We define

$$
G=\sum_{j=1}^{\infty}\left(\sum_{i=1}^{\infty} \alpha_{i j}\left(\cdot, e_{i}\right)\right) e_{j},
$$

where $\left\{e_{n}\right\}$ is an orthonormal basis of $H$ and $\left\{\alpha_{i j}\right\}$ is a family of nonzero complex numbers restrained by $\sum_{j=1}^{\infty} \sum_{i=1}^{\infty}\left|\alpha_{i j}\right|^{2}<+\infty$.

It is proved in [2, p. 393] that the strong closure of all finite rank operators in a nest of algebra $A \mathscr{N}$ is equal to $A \mathscr{N}$. Motivated by [1, p. 1], we shall show that $B(H)$ is the strong-principal bimodule of $R_{\left\{e_{n}\right\}}^{1} A \mathscr{N}$ with respect to $G$ for a certain orthonormal basis $\left\{e_{n}\right\}$ of $H$, that is, the representation is possible as long as we substitute the basic units $x \otimes y$ of the finite rank operators in the nest algebra by the new basic units $\left(e_{i} \otimes e_{j}\right) G\left(e_{k} \otimes e_{l}\right)$, where $e_{i} \otimes e_{j}$ and $e_{k} \otimes e_{l}$ are rank-1 operators in the nest algebra and are composed only by the elements of a certain orthonormal basis $\left\{e_{n}\right\}$ of $H$.

$$
\text { 1. } R_{\left\{e_{n}\right\}}^{1} A \mathscr{N}
$$

Lemma 1.1. (Ringrose, [5, p. 64]). Let $\mathcal{N}$ be a complete nest, and let $x$ and $y$ be nonzero vectors in $H$. Then $x \otimes y \in A \mathscr{N}$ if and only if there is an $N \in \mathcal{N}$ such that $x \in\left(N_{-}\right)^{\perp}, y \in N$.

The above lemma can be written as

$$
R^{1} A \mathcal{N}=\left\{x \otimes y: x \in\left(N_{-}\right)^{\perp}, y \in N, N \in \mathscr{N}\right\}
$$

If $\left\{e_{n}\right\}$ is an orthonormal basis in $H$, we have

$$
R_{\left\{e_{n}\right\}}^{1} A \mathscr{N}=\left\{e_{i} \otimes e_{j}: e_{i} \in\left(N_{-}\right)^{\perp}, e_{j} \in N ; e_{i}, e_{j} \in\left\{e_{n}\right\} ; N \in \mathscr{N}\right\}
$$

Lemma 1.2. Let $\mathcal{N}$ be a complete nest. Then there exists an orthonormal basis $\left\{e_{n}\right\}$ of $H$ with the property that for any $e_{i} \in\left\{e_{n}\right\}$ there exists $e_{j}, e_{k} \in\left\{e_{n}\right\}$ such that

$$
e_{j} \otimes e_{i}, e_{i} \otimes e_{k} \in A \mathscr{N}
$$

Proof. (i) $\{0\} \subset\{0\}_{+}$and $H_{-} \subset H$. Observe that $\left(\{0\}_{+}\right)_{-}=\{0\}$ and $H=$ $\{0\}^{\perp}=\left(\left(\{0\}_{+}\right)_{-}\right)^{\perp}$. Let $B_{1}, B_{2}$, and $B_{3}$ denote orthonormal bases of $\{0\}_{+}$, $H_{-} \ominus\{0\}_{+}$and $\left(H_{-}\right)^{\perp}$ respectively. We define $\left\{e_{n}\right\}=B_{1} \cup B_{2} \cup B_{3}$. For any $e_{i} \in\left\{e_{n}\right\}$, if $e_{i} \in B_{1}$, it follows from $e_{i} \in H=\left(\left(\{0\}_{+}\right)_{-}\right)^{\perp}, e_{i} \in\{0\}_{+}$that $e_{i}$ 
can act as $e_{j}$ and $e_{k}$ as well; If $e_{i} \in B_{2}$, for any $e_{j} \in B_{3}$, it follows from $e_{j} \in\left(H_{-}\right)^{\perp}, e_{i} \in H$ that $e_{j} \otimes e_{i} \in A \mathscr{N}$, and for any $e_{k} \in B_{1}$, it follows from $e_{i} \in H=\left(\left(\{0\}_{+}\right)_{-}\right)^{\perp}, e_{k} \in\{0\}_{+}$that $e_{i} \otimes e_{k} \in A \mathscr{N}$; If $e_{i} \in B_{3}$, it follows from $e_{i} \in\left(H_{-}\right)^{\perp}, e_{i} \in H$ that $e_{i}$ can act as $e_{j}$ and $e_{k}$ as well.

(ii) $\{0\}=\{0\}_{+}$and $H_{-}=H$. There exists a sequence $\left\{N_{n}\right\}_{n=-\infty}^{+\infty}$ in $\mathscr{N}$ satisfying (i) if $i<j$, then $N_{i} \subset N_{j}$, (ii) $\lim _{n \rightarrow-\infty} P_{N_{n}}=0$ and $\lim _{n \rightarrow+\infty} P_{N_{n}}=$ $I$ all in the strong operator topology $\left[1\right.$, p. 2]. Let $B_{n}$ denote an orthonormal basis of $N_{n} \ominus N_{n-1}$ for each $n$. We define $\left\{e_{n}\right\}=\bigcup_{n=-\infty}^{+\infty} B_{n}$. For any $e_{i} \in$ $\left\{e_{n}\right\}$, if $e_{i} \in B_{n}$, then any vector in $B_{m}(m>n)$ can act as $e_{j}$, while any vector in $B_{m}(m<n)$ can act as $e_{k}$.

(iii) The proof for the case either $\{0\} \subset\{0\}_{+}$and $H_{-}=H$ or $\{0\}=\{0\}_{+}$ and $H_{-} \subset H$ is trivial.

Lemma 1.3. Let $\mathcal{N}$ be a complete nest. Then there exists an orthonormal basis $\left\{e_{n}\right\}$ of $H$ with the property that for any $e_{i}, e_{j} \in\left\{e_{n}\right\}$ there is a complex number $\alpha(i, j) \neq 0$ such that

$$
\alpha(i, j)\left(e_{i} \otimes e_{j}\right) \in\left(R_{\left\{e_{n}\right\}}^{1} A \mathscr{N}\right) G\left(R_{\left\{e_{n}\right\}}^{1} A \mathscr{N}\right) .
$$

Proof. Let $\left\{e_{n}\right\}$ be the orthonormal basis of $H$ defined in Lemma 1.2. For any $e_{i}, e_{j} \in\left\{e_{n}\right\}$, we can find $e_{i_{0}}, e_{j_{0}} \in\left\{e_{n}\right\}$ such that $e_{i} \otimes e_{i_{0}}, e_{j_{0}} \otimes e_{j} \in A \mathcal{N}$.

$$
\begin{aligned}
e_{i} \otimes e_{j} & =\frac{1}{\alpha_{i_{0} j_{0}}}\left(G e_{i_{0}}, e_{j_{0}}\right) e_{i} \otimes e_{j} \\
& =\frac{1}{\alpha_{i_{0} j_{0}}}\left(e_{j_{0}} \otimes e_{j}\right)\left(e_{i} \otimes G e_{i_{0}}\right)=\frac{1}{\alpha_{i_{0} j_{0}}}\left(e_{j_{0}} \otimes e_{j}\right) G\left(e_{i} \otimes e_{i_{0}}\right), \\
\alpha_{i_{0} j_{0}} e_{i} \otimes e_{j} & =\left(e_{j_{0}} \otimes e_{j}\right) G\left(e_{i} \otimes e_{i_{0}}\right) \in\left(R_{\left\{e_{n}\right\}}^{1} A \mathscr{N}\right) G\left(R_{\left\{e_{n}\right\}}^{1} A \mathcal{N}\right) .
\end{aligned}
$$

Theorem 1.4. Let $\mathcal{N}$ be a complete nest. Then there exists an orthonormal basis $\left\{e_{n}\right\}$ of $H$ such that

$$
B(H)=\left[\left(R_{\left\{e_{n}\right\}}^{1} A \mathscr{N}\right) G\left(R_{\left\{e_{n}\right\}}^{1} A \mathscr{N}\right)\right]^{(S)} .
$$

Proof. Let $\left\{e_{n}\right\}$ be the orthonormal basis of $H$ defined in Lemma 1.2. We know that

$$
B(H)=\left[R^{1}(H)\right]^{(S)} .
$$

For any $x, y \in H$, let $x=\sum_{i=1} \alpha_{i} e_{i}, y=\sum_{j=1} \beta_{j} e_{j} \cdot \sum_{j=1}^{n} \sum_{i=1}^{n} \alpha_{i} \beta_{j} e_{i} \otimes e_{j}$ converges in the norm operator topology to $x \otimes y[6$, p. 8$]$, so we have

$$
\left[R^{1}(H)\right]^{(S)}=\left[R^{1}\left(\left\{e_{n}\right\}\right)\right]^{(S)} .
$$

By Lemma 1.3, we have

$$
\left[R^{1}\left(\left\{e_{n}\right\}\right)\right]^{(S)} \subseteq\left[\left(R_{\left\{e_{n}\right\}}^{1} A \mathscr{N}\right) G\left(R_{\left\{e_{n}\right\}}^{1} A \mathscr{N}\right)\right]^{(S)} \subseteq B(H)
$$

Therefore,

$$
B(H)=\left[\left(R_{\left\{e_{n}\right\}}^{1} A \mathscr{N}\right) G\left(R_{\left\{e_{n}\right\}}^{1} A \mathscr{N}\right)\right]^{(S)} .
$$

Theorem 1.5. Let $\mathcal{N}$ be a complete nest. Then there exists an orthonormal basis $\left\{e_{n}\right\}$ of $H$ such that

$$
K(H)=\left[\left(R_{\left\{e_{n}\right\}}^{1} A \mathscr{N}\right) G\left(R_{\left\{e_{n}\right\}}^{1} A \mathscr{N}\right)\right]^{(n)} .
$$


Proof. Let $\left\{e_{n}\right\}$ be the orthonormal basis of $H$ defined in Lemma 1.2. We know that

$$
K(H)=\left[R^{1}(H)\right]^{(n)} \quad[6, \text { p. } 18] .
$$

By the proof of Theorem 1.4, we have

$$
\left[R^{1}(H)\right]^{(n)}=\left[R^{1}\left(\left\{e_{n}\right\}\right)\right]^{(n)} .
$$

By Lemma 1.3, we have

$$
\left[R^{1}\left(\left\{e_{n}\right\}\right)\right]^{(n)} \subseteq\left[\left(R_{\left\{e_{n}\right\}}^{1} A \mathscr{N}\right) G\left(R_{\left\{e_{n}\right\}}^{1} A \mathscr{N}\right)\right]^{(n)} \subseteq K(H)
$$

Therefore, $K(H)=\left[\left(R_{\left\{e_{n}\right\}}^{1} A \mathscr{N}\right) G\left(R_{\left\{e_{n}\right\}}^{1} A \mathscr{N}\right)\right]^{(n)}$.

$$
\text { 2. } R_{\left\{e_{n}\right\}}^{1} R A \mathscr{N} \text { AND } R_{\left\{e_{n}\right\}}^{1} R^{\infty} A \mathscr{N}
$$

Lemma 2.1. Let $\mathcal{N}$ be a complete nest, and let $x$ and $y$ be nonzero vectors in $H$. Then $x \otimes y \in R A \mathscr{N}\left(R^{\infty} A \mathscr{N}\right)$ if and only if there is $N \in \mathscr{N}$ such that $x \in N^{\perp}, y \in N$.

Proof. Let $x$ and $y$ be nonzero vectors in $H$. If there is $N \in \mathscr{N}$ such that $x \in N^{\perp}, y \in N$, by the corollary in $\S 0$, it follows from

$$
\begin{aligned}
x \otimes y & =(\cdot, x) y=\left(\cdot, P_{N^{\perp}} x\right) P_{N} y \\
& =\left(P_{N^{\perp}} \cdot, x\right) P_{N} y=P_{N}(x \otimes y) P_{N^{\perp}}
\end{aligned}
$$

that $x \otimes y$ is in $R A \mathscr{N}\left(R^{\infty} A \mathscr{N}\right)$.

Now let $x \otimes y \in R A \mathscr{N}\left(R^{\infty} A \mathscr{N}\right)$. Since $x \otimes y \in A \mathscr{N}$, there exists $N \in \mathscr{N}$ such that $x \in\left(N_{-}\right)^{\perp}, y \in N$, and since $x \otimes y \in R A \mathscr{N}\left(R^{\infty} A \mathscr{N}\right)$, given $\varepsilon>0$ there is a finite (perhaps infinite) $\mathscr{N}$-partition $\left\{E_{n}\right\}$ such that $\left\|P_{E_{n}}(x \otimes y) P_{E_{n}}\right\|<$ $\varepsilon$ for all $n$. Observe that because $N \ominus N_{-}$is a minimal interval, there exist some $E_{n} \supseteq N \ominus N_{-}$, and it follows from

$$
\left\|P_{N \ominus N-}(x \otimes y) P_{N \ominus N-}\right\| \leq\left\|P_{E_{n}}(x \otimes y) P_{E_{n}}\right\|<\varepsilon
$$

that

$$
P_{N \ominus N-}(x \otimes y) P_{N \ominus N-}=0
$$

so

$$
\left(P_{N \ominus N-} \cdot, x\right) P_{N \ominus N-} y=0 .
$$

Assuming that $N \subset N$ and $y \bar{\epsilon} N$, we have $\left(P_{N \ominus N-} \cdot, x\right)=0$, i.e. , $x \perp$ $N \ominus N_{-}$. Together with $x \perp N_{-}$, thus we have $x \in N^{\perp}$.

The above lemma can be written as

$$
R^{1} R A \mathscr{N}=R^{1} R^{\infty} A \mathscr{N}=\left\{x \otimes y: x \in N^{\perp}, y \in N ; N \in \mathscr{N}\right\}
$$

If $\left\{e_{n}\right\}$ is an orthonormal basis of $H$, we have $R_{\left\{e_{n}\right\}}^{1} R A \mathscr{N}=R_{\left\{e_{n}\right\}}^{1} R^{\infty} A \mathscr{N}=\left\{e_{i} \otimes e_{j}: e_{i} \in N^{\perp}, e_{j} \in N ; e_{i}, e_{j} \in\left\{e_{n}\right\} ; N \in \mathscr{N}\right\}$

The proofs for the following Lemma 2.2, Lemma 2.3, and Theorem 2.4 are similar to those of case (ii) of Lemma 1.2, Lemma 1.3, and Theorem 1.4 and so are omitted. 
Lemma 2.2. Let $\mathcal{N}$ be a complete nest with $\{0\}=\{0\}_{+}$and $H_{-}=H$. Then there exists an orthonormal basis $\left\{e_{n}\right\}$ of $H$ with the property that for any $e_{i} \in\left\{e_{n}\right\}$, there exist $e_{j}, e_{k} \in\left\{e_{n}\right\}$ such that $e_{j} \otimes e_{i}, e_{i} \otimes e_{k} \in R A \mathscr{N}$.

Lemma 2.3. Let $\mathscr{N}$ be a complete nest with $\{0\}=\{0\}_{+}$and $H_{-}=H$. Then there exists an orthonormal basis $\left\{e_{n}\right\}$ of $H$ with the property that for any $e_{i}, e_{j} \in\left\{e_{n}\right\}$, there exists a complex number $\alpha(i, j) \neq 0$ such that

$$
\alpha(i, j)\left(e_{i} \otimes e_{j}\right) \in(R A \mathscr{N}) G(R A \mathscr{N}) .
$$

Theorem 2.4. Let $\mathscr{N}$ be a complete nest with $\{0\}=\{0\}_{+}$and $H_{-}=H$. Then there exists an orthonormal basis $\left\{e_{n}\right\}$ of $H$ such that

$$
B(H)=\left[\left(R_{\left\{e_{n}\right\}}^{1} R A \mathscr{N}\right) G\left(R_{\left\{e_{n}\right\}}^{1} R A \mathscr{N}\right)\right]^{(S)} .
$$

Theorem 2.5. Let $\mathcal{N}$ be a complete nest with either $\{0\} \subset\{0\}_{+}$or $H_{-} \subset H$. Then for any $G \in B(H)$, neither

$$
B(H)=[(R A \mathscr{N}) G(R A \mathscr{N})]^{(S)}
$$

nor

$$
K(H)=[(R A \mathscr{N}) G(R A \mathscr{N})]^{(n)}
$$

is true.

Proof. If $\{0\} \subset\{0\}_{+}$, then by Lemma 2.1, we have

$$
R A \mathscr{N} \subseteq\left\{f \otimes e: f \in\left(\{0\}_{+}\right)^{\perp}, e \in H\right\} .
$$

For any $f_{1}, f_{2} \in\left(\{0\}_{+}\right)^{\perp}$ and $e_{1}, e_{2} \in H$,

$$
\left(f_{1} \otimes e_{1}\right) G\left(f_{2} \otimes e_{2}\right)\{0\}_{+}=\left(G e_{2}, f_{1}\right)\left(f_{2} \otimes e_{1}\right)\{0\}_{+}=\{0\} .
$$

Thus, evidently, for any nonzero vector $e$ in $\{0\}_{+}$

$$
e \otimes e \bar{\epsilon}[(R A \mathscr{N}) G(R A \mathscr{N})]^{(S)} .
$$

If $H_{-} \subset H$, then by Lemma 2.1, we have

$$
R A \mathscr{N} \subseteq\left\{e \otimes g: e \in H, g \in H_{-}\right\} .
$$

For any $e_{1}, e_{2} \in H$ and $g_{1}, g_{2} \in H_{-}$

$$
\operatorname{ran}\left(e_{1} \otimes g_{1}\right) G\left(e_{2} \otimes g_{2}\right)=\operatorname{ran}\left(G g_{2}, e_{1}\right)\left(e_{2} \otimes g_{1}\right) \subseteq H_{-} .
$$

Thus, evidently, for any nonzero vector $f \in\left(H_{-}\right)^{\perp}$,

$$
f \otimes f \bar{\epsilon}[(R A \mathscr{N}) G(R A \mathscr{N})]^{(S)} .
$$

In view of the compactness of $e \otimes e$ and $f \otimes f$, neither of the expressions in the theorem is valid.

\section{REFERENCES}

1. Xingde, Dai, Norm-principle bimodules of nest algebras, J. Funct. Anal. 90 (1990), 369-390.

2. J. A. Erdös, Operators of finite rank in nest algebras, J. London Math. Soc. 43 (1968), 391-397.

3. D. R. Larson, Nest algebras and similarity transformations, Ann. of Math. 121 (1985), 409-427.

4. __, , Triangularity in operator algebras, Pitman Research Notes in Math., no. 192, Longman, Whiteplains, NY, pp. 121-188. 
5. J. R. Ringrose, On some algebras of operators, Proc. London Math. Soc. (3) 15 (1965), 61-83.

6. Robert Schatten, Norm ideals of completely continuous operators, Springer-Verlag, Berlin, Gottinggen and Heidelberg, 1960.

Department of Mathematics, Qufu Normal University, Qufu, Shandong, 273165, PeoPLE'S REPUBLIC OF CHINA 